\title{
Effects of Different Levels of Pressure Support on Intra-Individual Breath-to-Breath Variability
}

\author{
Monica R Cruz MSc, Luciana M Camilo PhD, Luis Felipe SC Paula MSc, \\ Andre M Japiassú MD PhD, Alessandro Beda PhD, Alysson R Carvalho PhD, \\ Fernando A Bozza MD PhD, and Denise M Medeiros MD PhD
}

\begin{abstract}
BACKGROUND: Evidence exists that during pressure support ventilation (PSV), the addition of an extrinsic (ie, ventilator-generated) breath-to-breath variability (BBV) of breathing pattern improves respiratory function. If $\mathrm{BBV}$ is beneficial per se, choosing the PS level that maximizes it could be considered a valid strategy for conventional PSV. In this study, we evaluated the effect of different PS levels on intrinsic BBV in acutely ill, mechanically ventilated subjects to determine whether a significant relationship exists between PS level and BBV magnitude. METHODS: Fourteen invasively mechanically ventilated subjects were prospectively studied. PS was adjusted at $20 \mathrm{~cm} \mathrm{H} \mathrm{H}_{2} \mathrm{O}$ and sequentially reduced to 15,10 , and $5 \mathrm{~cm} \mathrm{H}_{2} \mathrm{O}$. Arterial blood gas analysis and pressure at $0.1 \mathrm{~s}$ after the onset of inspiration $\left(\mathbf{P}_{0.1}\right)$ were measured at each PS level. Airway and esophageal pressure and air flow were continuously recorded. Peak inspiratory flow, tidal volume $\left(\mathrm{V}_{\mathrm{T}}\right)$, breathing frequency, and pressure-time product (PTP) were calculated on a breath-by-breath basis. The breathing pattern variability was assessed by the coefficient of variation of the time series of $V_{T}$, peak inspiratory flow, and breathing frequency from $\sim 60$ consecutive breath cycles at each PS level. A general linear model for repeated measures was applied, with PS as an independent factor. A significance level of .05 was considered. RESULTS: Despite a large inter-individual difference in all measured variables $(P<.001)$, the coefficient of variation was as low as $30 \%$, and no significant differences in the coefficient of variation of peak inspiratory flow, breathing frequency, and $V_{T}$ between PS levels were observed $(P>.15)$. Additionally, a significant increase in $P_{0.1}$, PTP, and breathing frequency $(P<.01)$ and a reduction in $\mathrm{V}_{\mathrm{T}}(P<.001)$ were observed with $P S$ reduction. CONCLUSIONS: Despite a significant increase in spontaneous activity with PS reduction, BBV was not influenced by the PS level and was as low as $30 \%$ for all evaluated parameters. Key words: pressure support ventilation; breath-to-breath variability; patient-ventilator interaction; coefficient of variation. [Respir Care 2014;59(12):1888 -1894. (c) 2014 Daedalus Enterprises]
\end{abstract}

\section{Introduction}

During controlled artificial ventilation, the breathing pattern is usually monotonous with constant minute ventila-

\footnotetext{
Ms Cruz, Drs Japiassú, Bozza, and Medeiros are affiliated with the Laboratory of Clinical Research in Intensive Care Medicine, National Institute of Infectious Disease, Oswaldo Cruz Foundation, Rio de Janeiro, Brazil. Ms Camilo, Mr Paula, and Dr Carvalho are affiliated with the Laboratory of Respiration Physiology, Carlos Chagas Filho Institute of Biophysics, Universidade Federal do Rio de Janeiro, Brazil. Dr Beda is affiliated with the Department of Electronics, Federal University of Minas Gerais, Belo Horizonte, Brazil. Dr Bozza is also affiliated with the D'Or Institute for Research and Education, Rio de Janeiro, Brazil.
}

tion, tidal volume $\left(\mathrm{V}_{\mathrm{T}}\right)$, and breathing frequency. ${ }^{1}$ The intrinsic (ie, patient-generated) breath-to-breath variability (BBV) of breathing pattern, controlled by multiple feedback loops as well as neural and chemical factors, is fre-

\footnotetext{
This study was supported by the Centers of Excellence Program (Programa de Apoio a Núcleos de Excelência-Ministério da Ciência e Tecnologia and Ministério da Ciência e Tecnologia-Fundação de Amparo à Pesquisa do Estado do Rio de Janeiro), the Brazilian Council for Scientific and Technological Development (Ministério da Ciência e Tecnologia/Conselho Nacional de Desenvolvimento Científico e Tecnológico), and the Rio de Janeiro State Research Foundation (Fundação de Amparo à Pesquisa do Estado do Rio de Janeiro). The authors have disclosed no conflicts of interest.
} 
quently suppressed by sedation and muscle paralysis in critically ill patients. ${ }^{2}$

The addition of an extrinsic (ie, ventilator-generated) BBV of breathing pattern during controlled mechanical ventilation has been shown to be beneficial in experimental lung injury as well as during anesthesia, ${ }^{1}$ improving gas exchange, respiratory system elastance, and intrapulmonary shunt. ${ }^{3}$ In a recent experimental study, pressure support ventilation (PSV) with extrinsic BBV in PS (so-called noisy PSV) also improved pulmonary function while reducing inflammation and structural damage. ${ }^{4}$ Notably, several authors reported that the use of conventional PSV (with fixed PS) is associated with a relevant intrinsic BBV. ${ }^{5}$ Hence, if $\mathrm{BBV}$ is beneficial per se as suggested by studies using extrinsic variability, maximizing the intrinsic variability could be considered a target for conventional PSV to be achieved by choosing the PS level that results in the greatest $\mathrm{BBV}$ intrinsically generated by the subject.

In this context, in this study, we performed a preliminary assessment of the effect of different PS levels on intrinsic BBV in acutely ill, mechanically ventilated patients. Our hypothesis is that BBV is not affected by changes in PS, independent of its levels in acutely ill, mechanically ventilated patients.

\section{Methods}

\section{Study Population}

We conducted a prospective interventional study in the ICU of the National Institute of Infectious Disease at the Oswaldo Cruz Foundation in Rio de Janeiro, Brazil, from March to October 2011. The institutional review board approved the protocol, and written informed consent was obtained from all patients or their legal representatives before study inclusion.

Patients admitted to the ICU for respiratory failure and requiring invasive mechanical ventilation were screened for the protocol when switched to a spontaneous PS mode of ventilation and achieving sedation levels corresponding to a Richmond Agitation-Sedation Scale score of between -2 and +2 . Inclusion criteria were $>18$ y of age, hemodynamic stability defined by mean arterial pressure of $>65 \mathrm{~mm} \mathrm{Hg}$, and norepinephrine dose of $<0.1 \mu \mathrm{g} / \mathrm{kg} /$ min. Mild sedation was allowed with morphine, midazo-

Correspondence: Denise M Medeiros MD PhD or Fernando A Bozza MD $\mathrm{PhD}$, Laboratory of Clinical Research in Intensive Care Medicine, National Institute of Infectious Disease, Fundação Oswaldo Cruz, Avenue Brasil 4365, 21040-360 Rio de Janeiro, Brazil. E-mail: denise. medeiros@ipec.fiocruz.br or bozza.fernando@gmail.com/fernando.bozza@ipec.fiocruz.br.

DOI: $10.4187 /$ respcare. 02853

\section{QUICK LOOK}

\section{Current knowledge}

Pressure support ventilation (PSV) provides a constant level of pressure with each patient-triggered breath. During PSV, the intrinsic variability in tidal volume $\left(\mathrm{V}_{\mathrm{T}}\right)$ is reduced with changes in minute ventilation accomplished through a change in breathing frequency.

\section{What this paper contributes to our knowledge}

During PSV, a decrease in the pressure setting from 20 to $5 \mathrm{~cm} \mathrm{H}_{2} \mathrm{O}$ in $5-\mathrm{cm} \mathrm{H}_{2} \mathrm{O}$ increments increased subject breathing frequency but did not impact breath-to-breath $\mathrm{V}_{\mathrm{T}}$ variability. The coefficient of variation of $\mathrm{V}_{\mathrm{T}}$ was $<30 \%$ in $90 \%$ of subjects.

lam, and/or dexmedetomidine, and this, as well as PEEP and $\mathrm{F}_{\mathrm{IO}_{2}}$, was set by the attending physician. These parameters were not changed throughout the protocol. Subjects were mechanically ventilated (PB840, Covidien, Mansfield, Massachusetts) using the PSV mode with a flow trigger of $2.5 \mathrm{~L} / \mathrm{min}$, rise time of $90 \%$, and cycling criteria on $25 \%$ of peak inspiratory flow.

\section{Study Protocol}

After recording baseline ventilation, PS was adjusted at $20 \mathrm{~cm} \mathrm{H}_{2} \mathrm{O}$ and sequentially reduced to 15,10 , and $5 \mathrm{~cm} \mathrm{H}_{2} \mathrm{O}$, with 10-min intervals ${ }^{6}$ between changes (Fig. 1). In case of poor tolerance of the protocol, we defined as interruption criteria: (1) decrease in oxygen saturation of $<90 \%$, (2) respiratory distress with tachypnea over 35 breaths/min, and (3) psychomotor agitation and need for increasing sedation.

\section{Data Acquisition}

Airway pressure was measured proximal to the artificial airway, and esophageal pressure was measured with an esophageal balloon catheter (SmartCath, Bicore Monitoring Systems, Irvine, California) positioned at the distal part of the esophagus using gas pressure transducers (163PC01D48, Honeywell, Richmond, Virginia). The position of the balloon was verified before the beginning of measurement by the occlusion maneuver during 3-4 consecutive spontaneous breaths. ${ }^{7}$ Air flow was measured with a pneumotachometer (Hamilton Medical, Reno, Nevada) positioned right after the Y-piece of the circuit and connected to a differential pressure transducer (176PC07HD2, Honeywell). 


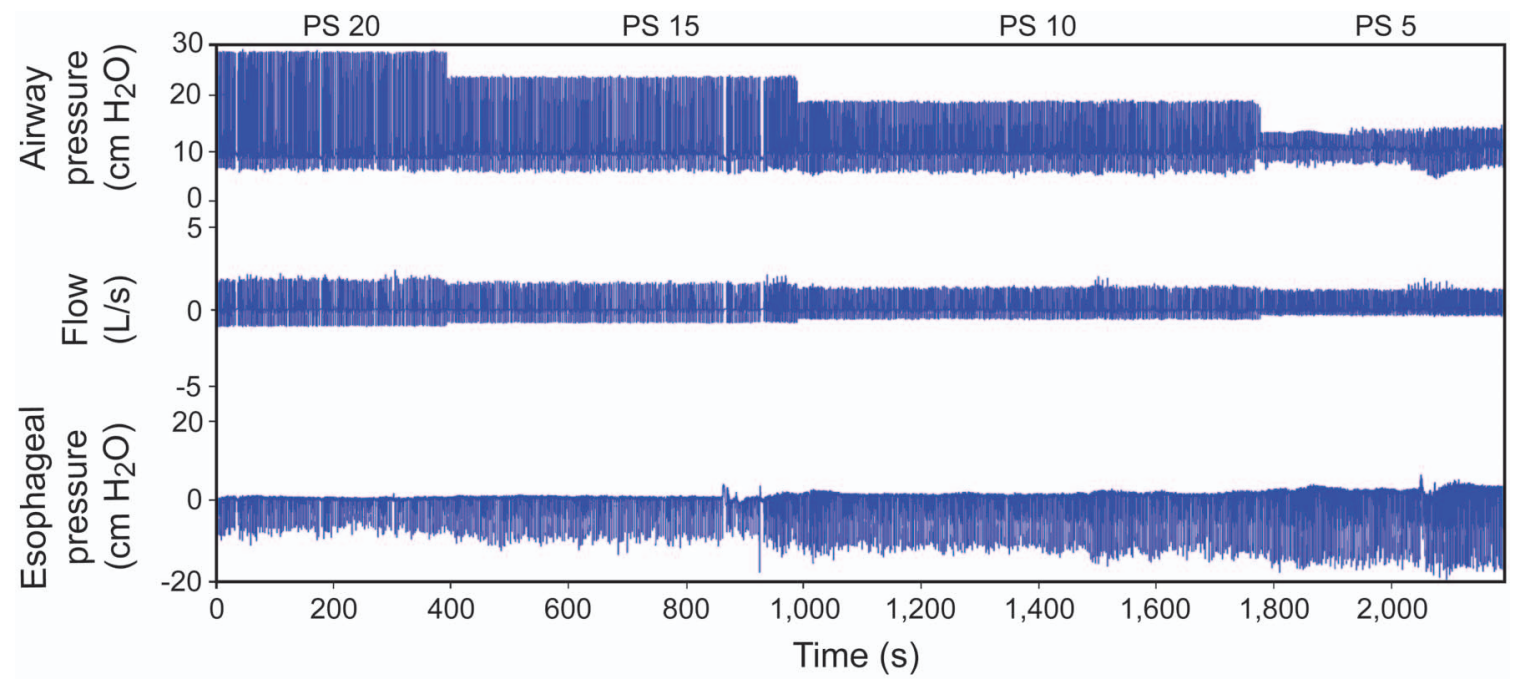

Fig. 1. Study protocol. Tracings from subject 13 showing continuous 2,180-s recordings of airway pressure, flow, and esophageal pressure during decremental pressure support (PS).

Airway pressure, esophageal pressure, air flow, mean arterial pressure, and electrocardiograms were continuously acquired with a sampling frequency of $1,000 \mathrm{~Hz}$ using custom-built data acquisition software and an analog-digital converter (NI 6008, National Instruments, Austin, Texas).

Before each change in PS, arterial blood was sampled for gas analysis (Stat Profile pHOx Plus L, Nova Biomedical, Waltham, Massachusetts) via a catheter previously inserted in the radial or femoral artery for mean arterial pressure monitoring.

\section{Data Processing}

Volume was calculated by the numerical integration of flow. Peak inspiratory flow, $\mathrm{V}_{\mathrm{T}}$, and breathing frequency were calculated for each respiratory cycle. The onset of inspiration and expiration was detected automatically as the points at which the flow signal crossed the zero value. Breathing frequency was calculated as the inverse of the interval between the onset of 2 consecutive inspirations. Airway pressure changes at $0.1 \mathrm{~s}$ from the onset of inspiration $\left(\mathrm{P}_{0.1}\right)$ were measured at the end of each step. The esophageal pressure-time product (PTP) was calculated during inspiration, taking the first value at the beginning of the respiratory cycle as the offset. ${ }^{8,9}$ Additionally, PTP was also corrected by breathing frequency for comparison with previous studies. ${ }^{10}$

The breathing pattern variability was assessed by the coefficient of variation ( $\mathrm{SD} /$ mean) of the time series of $\mathrm{V}_{\mathrm{T}}$, peak inspiratory flow, and breathing frequency for at least 3 min at the end of each PS level. ${ }^{11}$ Artifacts induced by cough, swallowing, and physical agitation of the subjects were excluded from our analysis. In case of artifacts, the record of the step could be prolonged. To measure ineffective triggers, a purpose-built routine (written in Matlab, Matworks, Natick, Massachusetts) was used to automatically identify the decrease in esophageal pressure without the occurrence of an effective inspiration on a breath-bybreath basis. A visual inspection was also performed after the automatic procedure.

\section{Statistical Analysis}

Statistical analysis was performed with Prism 4.0a (GraphPad Software, La Jolla, California) and Deducer 1.7-9 (Java GUI for R, http://www.deducer.org/ manual.html, Accessed July 10, 2014). The normality of the data (Shapiro-Wilk test) and the homogeneity of variances (Levene median test) were tested. If a null hypothesis failed to be rejected, a logarithmic transformation was applied, and after the null hypothesis was rejected, the test was repeated. All data are expressed as mean $\pm \mathrm{SD}$. The effects of PS levels and intra-individual variability in all measured parameters were assessed with a general linear model (with subjects and PS levels as factors). The significance level was set at .05 .

\section{Results}

Fourteen subjects were included; the main indications for mechanical ventilation were acute hypoxemic respiratory failure in 12 subjects and reduced level of consciousness in 2 subjects. Primary diagnoses, mechanical ventilation times before the study, PS levels before the study, $\mathrm{P}_{\mathrm{aO}_{2}} / \mathrm{F}_{\mathrm{IO}_{2}}$ indices at baseline, vasopressor use, PEEP, and Richmond Agitation-Sedation Scale scores of all subjects are shown in Table 1. Nine subjects were sedated during 
Table 1. Clinical Characteristics of Subjects

\begin{tabular}{|c|c|c|c|c|c|c|c|c|c|c|}
\hline Subject & $\begin{array}{c}\text { Age } \\
(\mathrm{y})\end{array}$ & $\begin{array}{c}\text { SAPS } \\
\text { II }\end{array}$ & $\begin{array}{l}\text { SOFA } \\
\text { Score }\end{array}$ & Vasopressors & $\mathrm{P}_{\mathrm{aO}_{2}} / \mathrm{F}_{\mathrm{IO}_{2}}$ & $\begin{array}{l}\text { Mechanical } \\
\text { Ventilation } \\
\text { Time (d) }\end{array}$ & $\begin{array}{l}\text { Baseline PS } \\
\left(\mathrm{cm} \mathrm{H}_{2} \mathrm{O}\right)\end{array}$ & $\begin{array}{c}\text { PEEP } \\
\left(\mathrm{cm} \mathrm{H}_{2} \mathrm{O}\right)\end{array}$ & Diagnosis & $\begin{array}{l}\text { RASS } \\
\text { Score }\end{array}$ \\
\hline 1 & 32 & 45 & 6 & No & 392 & 6 & 7 & 5 & Tuberculosis & 0 \\
\hline 2 & 30 & 21 & 9 & No & 383 & 2 & 9 & 5 & Urinary sepsis & -1 \\
\hline 3 & 34 & 21 & 7 & Yes & 267 & 10 & 14 & 10 & Pulmonary embolism & -1 \\
\hline 4 & 26 & 33 & 2 & No & 560 & 2 & 7 & 5 & Tuberculosis & 0 \\
\hline 5 & 39 & 66 & 9 & No & 498 & 5 & 10 & 5 & Meningitis & -2 \\
\hline 6 & 33 & 36 & 2 & Yes & 502 & 6 & 20 & 5 & Lung abscess & -2 \\
\hline 7 & 25 & 34 & 6 & Yes & 440 & 2 & 10 & 6 & Tuberculosis & -2 \\
\hline 8 & 42 & 48 & 8 & Yes & 271 & 3 & 16 & 8 & CAP & -2 \\
\hline 9 & 52 & 26 & 5 & No & 177 & 23 & 17 & 10 & CAP & -2 \\
\hline 10 & 67 & 36 & 5 & Yes & 416 & 6 & 15 & 6 & Urinary sepsis & +1 \\
\hline 11 & 45 & 63 & 12 & Yes & 200 & 10 & 15 & 5 & CAP & -2 \\
\hline 12 & 43 & 50 & 5 & No & 350 & 8 & 13 & 5 & CAP & +2 \\
\hline 13 & 41 & 45 & 7 & Yes & 355 & 7 & 10 & 5 & CAP & -1 \\
\hline 14 & 43 & 44 & 8 & Yes & 142 & 5 & 5 & 8 & Tuberculosis & -1 \\
\hline Mean \pm SD & $39 \pm 11$ & $40 \pm 13$ & $6.5 \pm 2.7$ & & $353 \pm 128$ & $6.7 \pm 5.3$ & $12 \pm 4.3$ & $6.3 \pm 1.8$ & & \\
\hline
\end{tabular}

SAPS II $=$ Simplified Acute Physiology Score II

SOFA $=$ Sequential Organ Failure Assessment

PS $=$ pressure support

RASS $=$ Richmond Agitation-Sedation Scale

$\mathrm{CAP}=$ community-acquired pneumonia

the study, and the maximum doses used were: morphine, $0.05 \mathrm{mg} / \mathrm{kg} / \mathrm{h}$; midazolam, $0.15 \mathrm{mg} / \mathrm{kg} / \mathrm{h}$; and dexmedetomidine, $0.5 \mu \mathrm{g} / \mathrm{kg} / \mathrm{h}$.

\section{Effect of PS Level on Respiratory Variables, PTP, and $\mathbf{P}_{0.1}$}

During the sequential reduction of the PS level, $\mathrm{V}_{\mathrm{T}}$ progressively decreased from $0.65 \pm 0.20$ to $0.45 \pm 0.11 \mathrm{~L}$, and there was a significant increase in breathing frequency from $18 \pm 7$ to $24 \pm 8$ breaths/min $(P<.001)$ (Fig. 2$)$. No significant differences were observed in minute ventilation (average value of $10.4 \pm 2.1 \mathrm{~L} / \mathrm{min}, P=.29$ ). A significant increase in work of breathing was also observed since PTP increased from $150 \pm 220$ to $390 \pm 540 \mathrm{~cm} \mathrm{H} \mathrm{H}_{2} \mathrm{O} \times \mathrm{s} / \mathrm{min}$ $(P<.01)$, and $\mathrm{P}_{0.1}$ increased from $2 \pm 2$ to $6.5 \pm$ $3.7 \mathrm{~cm} \mathrm{H}_{2} \mathrm{O}(P<.001)$. No ineffective trigger was detected in any of the subjects.

\section{Effect of PS Level on Breathing Pattern Variability}

PS reduction did not significantly affect the coefficient of variation of the time series of $\mathrm{V}_{\mathrm{T}}$, peak inspiratory flow, breathing frequency, and inspiratory time $(P>.15)$ (Fig. $3)$. The coefficient of variation of $\mathrm{V}_{\mathrm{T}}$ was $<30 \%$ in 52 of the 56 measurements performed.

A considerable heterogeneity among subjects was found in the coefficient of variation of the parameters considered. For $\mathrm{V}_{\mathrm{T}}$, the coefficient of variation ranged from $2 \%$ (almost absent) to $37 \%$ (similar to that of spontaneously breathing, healthy subjects). For breathing frequency and peak inspiratory flow, the coefficient of variation varied from 6 to $43 \%$ and from 3 to $37 \%$ between subjects, respectively.

\section{Effect of PS Level on Gas Exchange, Heart Rate, and Mean Arterial Pressure}

No significant effects of PS level on mean arterial pressure, heart rate, $\mathrm{P}_{\mathrm{aO}}$, and $\mathrm{P}_{\mathrm{aCO}}$ were found as shown in Table 2. The mean coefficient of variation of heart rate and that of mean arterial pressure were $9 \pm 17 \%$ and $3.1 \pm 1.6 \%$, respectively, and did not change with PS reduction.

\section{Discussion}

The main findings of this study are as follows. (1) In this group of acutely ill, mechanically ventilated subjects, despite the low level of sedation, changes in PS did not result in significant changes in BBV even though they caused significant adaptations in terms of subject effort measured by PTP and $\mathrm{P}_{0.1}$. (2) The coefficient of variation of the time series of peak inspiratory flow, breathing frequency, and $\mathrm{V}_{\mathrm{T}}$ was generally lower than that reported in the literature during spontaneous breathing but was comparable to that in patients with slight-to-moderate hypoxia and in patients with acute respiratory failure under PSV. (3) Significant inter-individual variability exists in all re- 
A

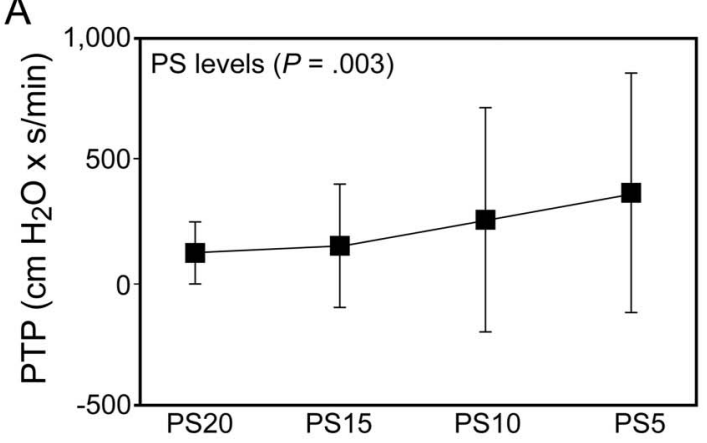

C

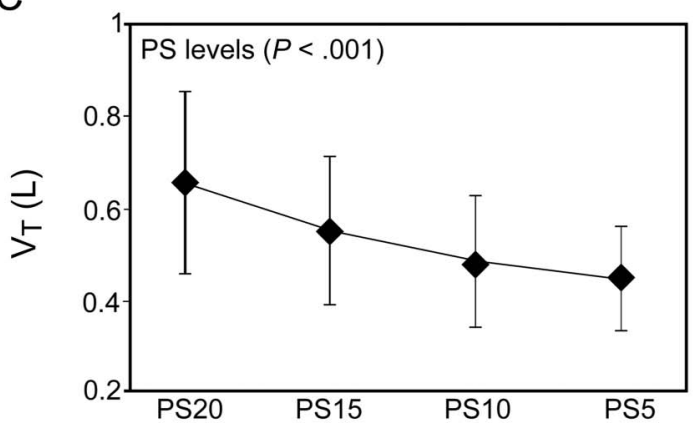

B

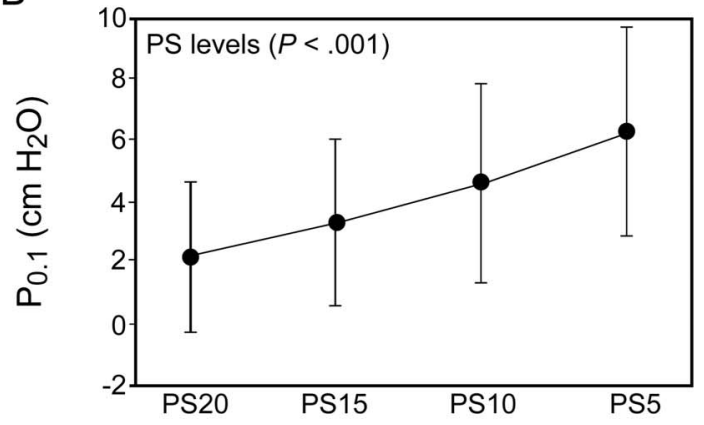

$\mathrm{D}$

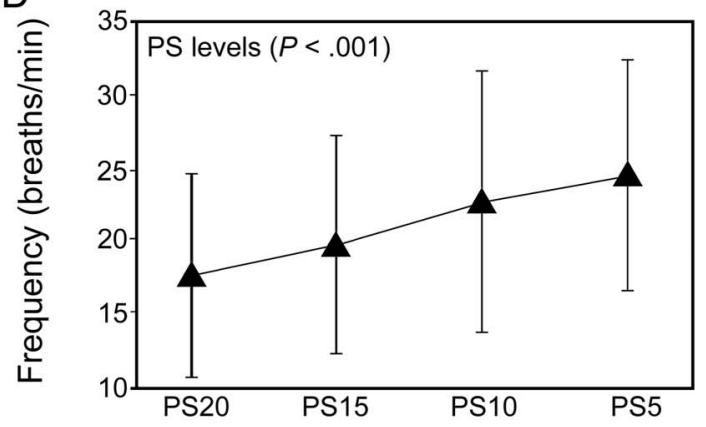

Fig. 2. Values of pressure-time product $(P T P)(A)$, respiratory drive $\left(P_{0.1}\right)(B)$, tidal volume $\left(V_{T}\right)(C)$, and breathing frequency $(D)$ at different levels of pressure support (PS). Data are shown as mean \pm SD.

A

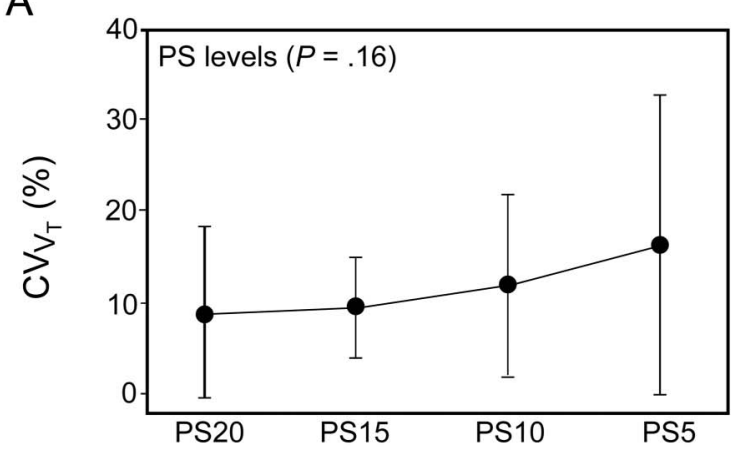

C

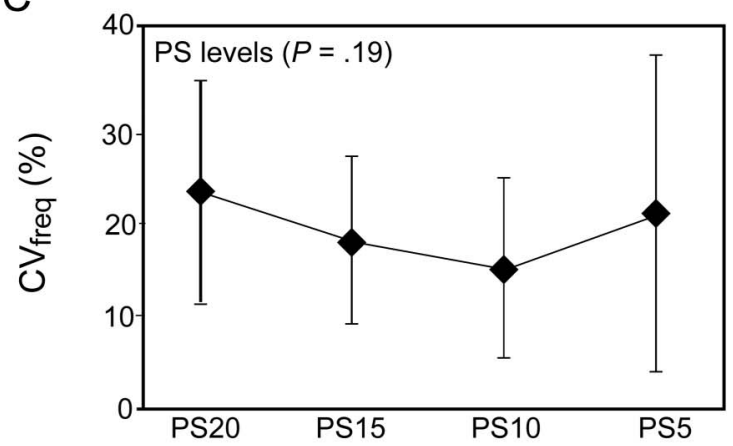

B

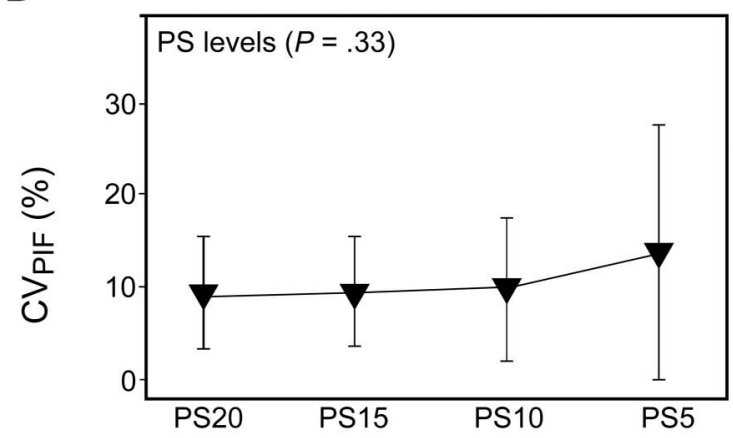

D

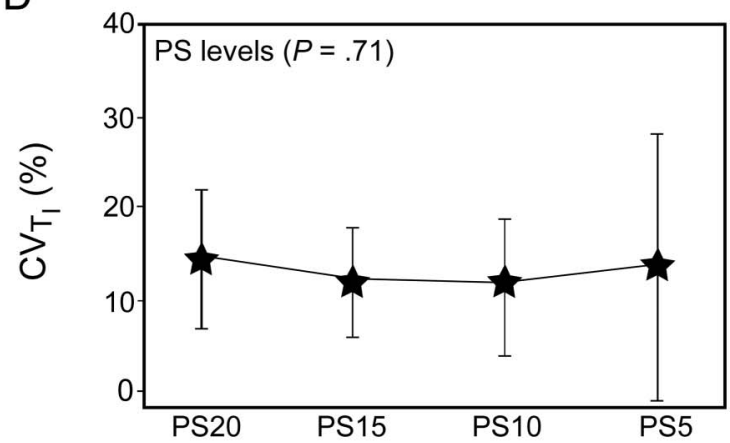

Fig. 3. Values of the coefficient of variation ( $S D /$ mean) of tidal volume $\left(C_{V_{T}}\right)(A)$, peak inspiratory flow $\left(C V_{P I F}\right)(B)$, breathing frequency $\left(C V_{\text {freq}}\right.$; breaths/min) $(C)$, and inspiratory time $\left(C_{T_{1}}\right)(D)$ at different levels of pressure support (PS). Data are presented as mean $\pm \mathrm{SD}$. 
Table 2. Analysis of Arterial Blood Gases and Hemodynamic Variables

\begin{tabular}{|c|c|c|c|c|c|}
\hline & \multicolumn{4}{|c|}{ Pressure Support } & \multirow{2}{*}{ PS Effect } \\
\hline & $20 \mathrm{~cm} \mathrm{H}_{2} \mathrm{O}$ & $15 \mathrm{~cm} \mathrm{H}_{2} \mathrm{O}$ & $10 \mathrm{~cm} \mathrm{H}_{2} \mathrm{O}$ & $5 \mathrm{~cm} \mathrm{H}_{2} \mathrm{O}$ & \\
\hline $\mathrm{pH}$ & $7.49 \pm 0.03$ & $7.48 \pm 0.03$ & $7.49 \pm 0.05$ & $7.49 \pm 0.06$ & .15 \\
\hline $\mathrm{P}_{\mathrm{aCO}_{2}}$ & $40.8 \pm 9$ & $42.5 \pm 9.1$ & $43.4 \pm 10.5$ & $42.4 \pm 10.5$ & .27 \\
\hline $\mathrm{P}_{\mathrm{aO}}$ & $131 \pm 50$ & $119.5 \pm 44.7$ & $120 \pm 36.9$ & $119.7 \pm 34.3$ & .44 \\
\hline Heart rate, beats/min & $91 \pm 18$ & $87.7 \pm 19.7$ & $88 \pm 20.3$ & $89.6 \pm 19.3$ & .14 \\
\hline Mean arterial pressure, $\mathrm{mm} \mathrm{Hg}$ & $97.6 \pm 14$ & $101.3 \pm 14$ & $102.2 \pm 12$ & $103.6 \pm 11$ & .06 \\
\hline
\end{tabular}

spiratory parameters, especially those related to BBV, suggesting that other factors seem to influence BBV beyond differences in ventilation. (4) To assure a certain level of BBV during PSV in a group of patients, the use of ventilator-generated variability seems necessary.

During assisted ventilation in critically ill patients, intrinsic BBV can be reduced depending on several factors, such as the mode of assisted ventilation, the level of sedation, and the severity of the underlying disease itself.

In the present study, we assessed the possible relationship between the mean level of PS and the amount of $\mathrm{BBV}$. A progressive reduction in PS resulted in considerable respiratory adaptations. In fact, PTP and $\mathrm{P}_{0.1}$ increased significantly, suggesting a progressively larger work load. However, BBV did not change significantly. Actually, if we assume that a reduction in the PS level increases respiratory work load, one would expect a reduction in the intrinsic BBV as a result of increased resistive or elastic loads, as reported previously in spontaneously breathing patients. ${ }^{12,13}$ Most likely, intrinsic mechanisms, such as respiratory drive or even the level of sedation, play a complex role in patient-ventilator interaction that might be more important than PS for the generation of BBV. ${ }^{14,15}$

The intra-individual breath-to-breath fluctuations in ventilatory variables, such as $\mathrm{V}_{\mathrm{T}}$, peak inspiratory flow, and breathing frequency, are well recognized and can be cyclically observed. ${ }^{16}$ Healthy subjects breathing spontaneously show a coefficient of variation of $\mathrm{V}_{\mathrm{T}}$ between $26 \%{ }^{17}$ and $33 \%,{ }^{18}$ which is reduced during lung disease. ${ }^{19}$ The coefficient of variation of $\mathrm{V}_{\mathrm{T}}$ during PSV in deeply sedated animals or patients with acute lung injury has been reported to be as low as $10 \% 18,20$ and up to $30 \%$ at a fixed PS level. In agreement with our results, a recent study reported the same level of intrinsic BBV, with a coefficient of variation of $\mathrm{V}_{\mathrm{T}}$ of $13.7 \pm 9.1 \%$ (mean $\pm \mathrm{SD}$ ), in critically ill patients ventilated with the PS mode. Interestingly, even intra-individual BBV in the present study was similar to that reported by Spieth et al. ${ }^{21}$

Increased variability in $\mathrm{V}_{\mathrm{T}}$ in critically ill patients has been associated with successful weaning from mechanical ventilation, ${ }^{18,22}$ and the coefficient of variation of $V_{T}$ has been proposed as a predictor for extubation outcome. ${ }^{18} \mathrm{In}$ this context, our results suggest that such a prediction is not influenced by the PS level and that very low PS levels can be avoided for this assessment, reducing the occurrence of fatigue and distress in the patient.

The results suggest that fine-tuning the PS is an effective strategy to obtain a level of BBV associated with potential improvements in gas exchange, lung mechanics, and lung pro-inflammatory mediators, as suggested in a previous study. ${ }^{4}$ From this point of view, the use of ventilator-generated variability seems indispensable. However, the interaction between intrinsically and extrinsically generated BBV is still unknown, and it is possible to speculate that some patients would maintain basically the same breathing pattern irrespective of the presence of BBV, undermining the expected benefits of this ventilation strategy. This possibility deserves further investigation to fully exploit extrinsic BBV in a broad clinical population.

\section{Limitations}

No randomization of the sequence of PS levels was applied in our protocol, which was planned to reduce the ventilatory support progressively. Hence, a sequence effect cannot be excluded, but it seems more likely that this could have induced, rather than suppressed, a dependence of respiratory variability on PS by inducing changes in variability secondary to loading or fatigue. Moreover, the severity of illness of our subjects may have influenced the $\mathrm{BBV}$, as described by other authors.

It is also possible that increased subject effort related to PS reduction promoted some level of anxiety, which is known to affect BBV considerably, ${ }^{16}$ possibly masking a relationship between BBV and PS levels. Although we maintained sedation control in our subjects at Richmond Agitation-Sedation Scale scores of between -2 and +2 , we cannot exclude that these drugs may still have some effect on BBV.

\section{Conclusions}

BBV during PSV was not influenced by the level of pressure assistance offered to the subject despite significant changes in $\mathrm{V}_{\mathrm{T}}$, breathing frequency, and PTP. The possible beneficial effects of an extrinsic BBV and its 


\section{Pressure Support and Breath-To-Breath Variability}

interaction with the intrinsic breath pattern variability in patients during PSV remain to be determined.

\section{REFERENCES}

1. Boker A, Haberman CJ, Girling L, Guzman RP, Louridas G, Tanner $\mathrm{JR}$, et al. Variable ventilation improves perioperative lung function in patients undergoing abdominal aortic aneurysmectomy. Anesthesiology 2004;100(3):608-616.

2. Mutch WA, Eschun GM, Kowalski SE, Graham MR, Girling LG, Lefevre GR. Biologically variable ventilation prevents deterioration of gas exchange during prolonged anaesthesia. Br Journal Anaesth 2000;84(2):197-203.

3. Boker A, Graham MR, Walley KR, McManus BM, Girling LG, Walker E, et al. Improved arterial oxygenation with biologically variable or fractal ventilation using low tidal volumes in a porcine model of acute respiratory distress syndrome. Am J Respir Crit Care Med 2002;165(4):456-462.

4. Spieth PM, Carvalho AR, Güldner A, Kasper M, Schubert R, Carvalho NC, et al. Pressure support improves oxygenation and lung protection compared to pressure-controlled ventilation and is further improved by random variation of pressure support. Crit Care Med 2011;39(4):746-755.

5. Giraldo BF, Chaparro JA, Caminal P, Benito S. Characterization of the respiratory pattern variability of patients with different pressure support levels. Conf Proc IEEE Eng Med Biol Soc 2013;2013:38493852.

6. Jubran A, Grant BJ, Laghi F, Parthasarathy S, Tobin MJ. Weaning prediction: esophageal pressure monitoring complements readiness testing. Am J Respir Crit Care Med 2005;171(11):1252-1259.

7. Baydur A, Behrakis PK, Zin WA, Jaeger M, Milic-Emili J. A simple method for assessing the validity of the esophageal balloon technique. Am Rev Respir Dis 1982;126(5):788-791.

8. Sassoon CS, Light RW, Lodia R, Sieck GC, Mahutte CK. Pressuretime product during continuous positive airway pressure, pressure support ventilation, and T-piece during weaning from mechanical ventilation. Am Rev Respir Dis 1991;143(3):469-475.

9. Jubran A, Van de Graaff WB, Tobin MJ. Variability of patientventilator interaction with pressure support ventilation in patients with chronic obstructive pulmonary disease. Am J Respir Crit Care Med 1995;152(1):129-136

10. Thille AW, Rodriguez P, Cabello B, Lellouche F, Brochard L. Patient-ventilator asynchrony during assisted mechanical ventilation. Intensive Care Med 2006;32(10):1515-1522.
11. Tobin MJ, Mador MJ, Guenther SM, Lodato RF, Sackner MA. Variability of resting respiratory drive and timing in healthy subjects. J Appl Physiol 1988;65(1):309-317.

12. Brack T, Jubran A, Tobin MJ. Effect of elastic loading on variational activity of breathing. Am J Respir Crit Care Med 1997;155(4):13411348.

13. Brack T, Jubran A, Tobin MJ. Effect of resistive loading on variational activity of breathing. Am J Respir Crit Care Med 1998;157(6 Pt 1):1756-1763.

14. Mitsis GD, Governo RJ, Rogers R, Pattinson KT. The effect of remifentanil on respiratory variability, evaluated with dynamic modeling. J Appl Physiol 2009;106(4):1038-1049.

15. Saponjic J, Radulovacki M, Carley DW. Respiratory pattern modulation by the pedunculopontine tegmental nucleus. Respir Physiol Neurobiol 2003;138(2-3):223-237.

16. Tobin MJ, Chadha TS, Jenouri G, Birch SJ, Gazeroglu HB, Sackner MA. Breathing patterns. 2. Diseased subjects. Chest 1983;84(3):286294

17. Kuratomi Y, Okazaki N, Ishihara T, Arai T, Kira S. Variability of breath-by-breath tidal volume and its characteristics in normal and diseased subjects. Ventilatory monitoring with electrical impedance pneumography. Jpn J Med 1985;24(2):141-149.

18. Bien MY, Hseu SS, Yien HW, Kuo BI, Lin YT, Wang JH, Kou YR. Breathing pattern variability: a weaning predictor in postoperative patients recovering from systemic inflammatory response syndrome. Intensive Care Med 2004;30(2):241-247.

19. Wysocki M, Cracco C, Teixeira A, Mercat A, Diehl JL, Lefort Y, et al. Reduced breathing variability as a predictor of unsuccessful patient separation from mechanical ventilation. Crit Care Med 2006; 34(8):2076-2083.

20. Gama de Abreu M, Spieth PM, Pelosi P, Carvalho AR, Walter C, Schreiber-Ferstl A, et al. Noisy pressure support ventilation: a pilot study on a new assisted ventilation mode in experimental lung injury. Crit Care Med 2008;36(3):818-827.

21. Spieth PM, Güldner A, Huhle R, Beda A, Bluth T, Schreiter D, et al. Short-term effects of noisy pressure support ventilation in patients with acute hypoxemic respiratory failure. Crit Care 2013;17(5):R261.

22. Bien MY, Shui Lin Y, Shih CH, Yang YL, Lin HW, Bai KJ, et al. Comparisons of predictive performance of breathing pattern variability measured during T-piece, automatic tube compensation, and pressure support ventilation for weaning intensive care unit patients from mechanical ventilation. Crit Care Med 2011;39(10):2253-2262. 